\title{
Using $\Delta$ - Discriminate Method to Determine the Stability and Bifurcation of Chen Chaotic System
}

\section{Saad Fawzi AL-Azzawi}

saad_fawzi78@yahoo.com

Department of Mathematics, College of

Computer Sciences and Mathematics,

University of Mosul, Mosul, Iraq
Received on: 15/03/2011

\section{ABSTRACT}

Karam A. Abed

Dar Ibn Al-Atheer for Printing and Publisher, University of Mosul, Mosul,
Iraq

Accepted on: 16/05/2011

The aim of this paper to found that the stability and bifurcation of Chen System by using a new method which called $\Delta$ - discriminate method as will as we found the stability at the second critical point $P_{+}(\sqrt{\beta(2 r-\sigma)}, \sqrt{\beta(2 r-\sigma)}, 2 r-\sigma)$ by another method, and we showed that the new method depended on the roots to determent the stability and bifurcation of this system while the previous methods depended on the parameters $\sigma, 2 r$, also we showed the method which used to find the stability at the second critical point depended on the critical value $\beta_{c}$ and parameter $\beta$. Finally, we get the same previous results but easily method.

Keywords: Chen system, stability, bifurcation, $\Delta$ - discriminate, Routh-Hurwitz.

$$
\begin{aligned}
& \text { استخدام طريقة ه } \Delta \text { - المميزة لتحديد الاستقرارية والتثعب لنظام Chen المضطرب }
\end{aligned}
$$

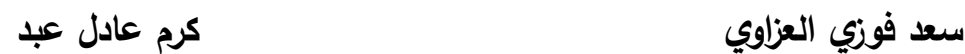

$$
\begin{aligned}
& \text { دار /بن الاثير للطباعة والنثر، جامعة الدوصل } \\
& \text { كلية علوم الحاسوب والرياضيات، جامعة الدوصل } \\
& \text { تاريخ قبول البحث: 2011/05/16 } \\
& \text { تاريخ استلام البحث: 2011/03/15 }
\end{aligned}
$$

\section{Introduction}

Chaos as a very interesting complex nonlinear phenomenon has been intensively studied in the last three decades within the science, mathematics and engineering communities [5].

In 1963, Lorenz discovered chaos in a simple system of three autonomous ordinary differential equations [7]. In 1999, Chen found a similar but not topological equivalent chaotic attractor to Lorenz's, which is now known to be the dual of the Lorenz system [4]. It is noticed that these systems can be classified into two types by the definition of Vanece and Celikovsky [3]: the Lorenz system satisfies the condition $a_{12} a_{21}>0$, the Chen system satisfies $a_{12} a_{21}<0$, where $a_{12}$ and $a_{21}$ are the corresponding elements in the linear part matrix $A=\left[a_{i j}\right]_{3 \times 3}$ of the system. 
The mathematical model of Lorenz system is a system of nonlinear ordinary differential equations which has the following form:

$$
\left.\begin{array}{l}
\dot{x}=\sigma(y-x) \\
\dot{y}=r x-x z-y \\
\dot{z}=x y-\beta z
\end{array}\right\}
$$

where $\sigma, r, \beta>o$, and Lorenz studied this system when $\sigma=10, r=28, \beta=8 / 3$. [3]

Chen system it also has the form of nonlinear ordinary differential equations:

$$
\left.\begin{array}{l}
\dot{x}=\sigma(y-x) \\
\dot{y}=(r-\sigma) x-x z+r y \\
\dot{z}=x y-\beta z
\end{array}\right\}
$$

where $\sigma, r, \beta>0$ and $r<\sigma<2 r$, Chen take $\sigma=35, r=28, \beta=3$. [6]

In the following we briefly describe some basic properties of the system (2).

\section{1- Symmetry and Invariance:}

Chen system has a symmetry $\mathrm{S}$ because the transformation:

$S:(x, y, z) \rightarrow(-x,-y, z)$

Which permits system invariant for all values of the system parameters $\sigma, r$ and $\beta$. Obviously, the z-axis itself is an orbit [7].

\section{2- Dissipative:}

The system (2) can be a dissipative, because the divergenence of the vector field, also called the trace of the Jacobian matrix is negative if and only if the sum of the parameters $\sigma, r$ and $\beta$ is positive

$$
\begin{aligned}
& \operatorname{div} \vec{V}=\frac{\partial \dot{x}}{\partial x}+\frac{\partial \dot{y}}{\partial y}+\frac{\partial \dot{z}}{\partial z}=\operatorname{Tr}(J)=-(\sigma-r+\beta), \\
& V(t)=V(0) e^{-(\sigma-r+\beta) t}
\end{aligned}
$$

So, the system will always be dissipative if and only if when $\sigma-r+\beta>0$ with

an exponential rate: $\frac{d \vec{V}}{d t}=e^{-(\sigma-r+\beta)}[8]$.

\section{3- Critical points:}

The critical points of system (2) can be easily found by solving the three equations $\dot{x}=\dot{y}=\dot{z}=0$, which lead to

$$
\begin{aligned}
& \sigma(y-x)=0, \\
& (r-\sigma) x-x z+r y=0, \\
& \text { and } x y-\beta z=0 .
\end{aligned}
$$

It can be easily verified that there are three critical points [3]:

$$
\begin{gathered}
p_{o}(0,0,0) \\
P_{+}(\sqrt{\beta(2 r-\sigma)}, \sqrt{\beta(2 r-\sigma)}, 2 r-\sigma) \\
P_{-}(-\sqrt{\beta(2 r-\sigma)},-\sqrt{\beta(2 r-\sigma)}, 2 r-\sigma)
\end{gathered}
$$


In $[1,4,5,7,10,11]$ studied the stability and bifurcation of Lorenz system, a new Lorenz-like system, a generalized Lorenz-like system, unified chaotic system, system derived from the Lorenz system, a new chaotic system respectively, by using Routh-Hurwitz method. Where we can conclusion that the real part of the roots $\lambda$ are negative if conditions of Routh-Hurwitz are satisfied without found the value of roots itself.

[8] studied the stability and bifurcation of Chen system by using Routh Hurwitz method where depended on the parameters $\sigma$ and $2 r$ to determine the stability at the point $P_{o}(0,0,0)$, while depened on the direct substitute for Routh-Hurwitz method to determine the stability at the point

$$
P_{ \pm}( \pm \sqrt{\beta(2 r-\sigma)}, \pm \sqrt{\beta(2 r-\sigma)}, 2 r-\sigma) \text {. }
$$

In this paper, we study the stability and bifurcation for the Chen system by using a new method which is called $\Delta$ - discriminate method. by this method we can find the exact roots of this system as well as we found the stability at the point $p_{ \pm}$by easily second method which depended on the critical value $\beta_{c}$, and we justified the same previous results, finally we showed that the relation between critical cases and bifurcation.

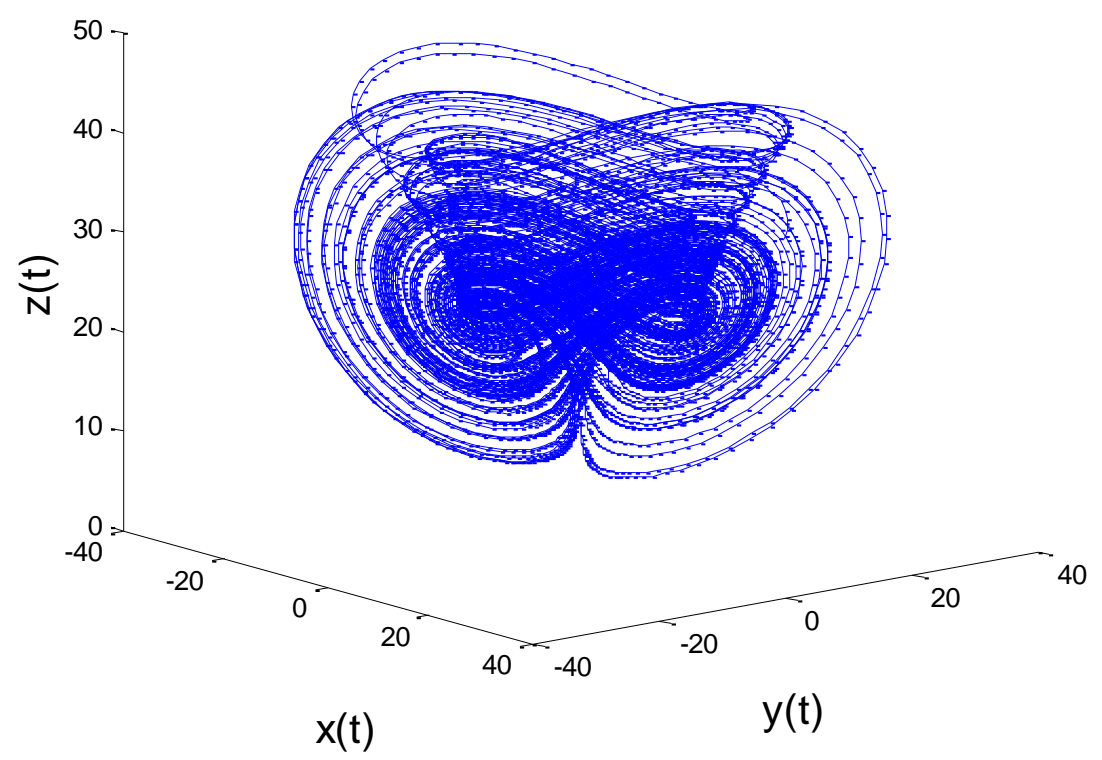

Figure 1: The attractor of Chen system

\section{2- Helping Results:}

In the context of ordinary differential equations ODEs the word "bifurcation" has come to mean any marked change in the structure of the orbits of a system (usually nonlinear) as a parameter passes through a critical value[2].

The theory of bifurcations of parameterized dynamical system is well known. One consider a vector field

$$
\dot{x}=f_{\mu}(x) \quad \mu \in R, \quad x \in R^{n}
$$


Depending on a parameter $\mu$ the critical point of the vector field are those $x_{0}, \mu_{0}$ such that $f_{\mu 0}\left(x_{0}\right)=0$.

Perhaps the most important property of critical point is its stability. In the first approximation, which is determined by stability of its liberalized system around $x_{0}, \mu_{0}$

$\dot{x}=D f_{\mu 0}\left(x_{0}\right) \quad \mu_{0} \in R, \quad x_{0} \in R^{n}$

Where $D f_{\mu 0}\left(x_{0}\right)$ is the Jacobian matrix of $f$.

\section{Theorem 1: (Hopf Bifurcation Theorem) [1]}

Suppose that the system $\dot{x}=f_{\mu}(x), \mu \in R, x \in R^{n}$ has critical point $\left(x_{0}, \mu_{0}\right)$, then this system has a Hopf bifurcation if the following properties are satisfied:

1- $D f_{\mu 0}\left(x_{0}\right)$ has a simple pair of pure imaginary eigenvalues and no other

eigenvalues with zero real parts.

$2-\left.\frac{d}{d \mu}\left(\operatorname{Re}\left(\lambda_{2 / 3}(\mu)\right)\right)\right|_{\mu=\mu 0}=d \neq 0$

\section{Remark 1: [1]}

Let $\lambda^{3}+a \lambda^{2}+b \lambda+c=0$ be the characteristic equation for a three-component system, where $a, c$ indicate the trace and determinant rest, then a hopf bifurcation takes place of the transit through the surface

$$
a b-c=0 \text { if } a, b, c>0
$$

This condition is a necessary condition for a hopf bifurcation.

\section{Remark 2: (Routh- Hurwitz Test) [2]}

All the roots of the indicated polynomial have negative real parts precisely when the given conditions are met.

$$
\lambda^{3}+a \lambda^{2}+b \lambda+c: \quad a>0, c>0, a b-c>0 .
$$

\section{Remark 3: (Critical Cases) [2]}

Critical cases in the theory of stability for differential equation means, that cases when the real part of all roots of the characteristic equation are non positive with the real part of at least one root being zero, other express which is neither stable nor unstable.

\section{Remark 4: [8]}

(i) if $\sigma>2 r$, then system (2) has only one critical point, $p_{o}(0,0,0)$;

(ii) if $\sigma<2 r$, then system (2) has three critical point: $p_{o}(0,0,0)$,

$$
p_{ \pm}( \pm \sqrt{\beta(2 r-\sigma)}, \pm \sqrt{\beta(2 r-\sigma)}, 2 \sigma-r)
$$

\section{Theorem 2: [8]}

(i) The solution of system (2) at critical point $p_{0}$ has the following cases:

1) Asymptotically stable if $\sigma>2 r$,

2) Unstable if $\sigma<2 r$.

(ii) The solution of system (2) at critical point $p_{+}$or $p_{-}$has the following cases: 
1) Asymptotically stable if $(\beta+\sigma-r) \beta r-2 \sigma \beta(2 r-\sigma)>0$,

2) Unstable if $(\beta+\sigma-r) \beta r-2 \sigma \beta(2 r-\sigma)<0$.

\section{Remark 5: (Critical Value) [8]}

Chen system has critical value which is $\sigma=2 r$ at origin and $\beta_{c}=\frac{\left(3 \sigma r-2 \sigma^{2}+r^{2}\right)}{r}$ at the critical point $p_{+}$.

\section{Remark 6: (Pitchfork Bifurcation and Hopf Bifurcation) [8]}

1-) Chen system has pitchfork bifurcation if $\sigma=2 r$.

When $\sigma=2 r$, this system has a one root being zero while the other two roots have a nonzero real part. In this case the solutions are $\lambda_{1}=-\beta, \lambda_{2}=0, \lambda_{3}<0$. and pitchfork bifurcation may appear only at the critical points $p_{0}$.

2-) Chen system has hopf bifurcation if $\beta=\beta_{c}$ and $r>(\sqrt{17}-3) \sigma / 2$.

When $\beta=\beta_{c}$, this system has is purely imaginary roots. In this case the solutions are $\lambda_{1}=2 \sigma(\sigma-2 r) / r, \lambda_{2,3}= \pm i \sqrt{\beta_{c} c}$ and hopf bifurcation may appear only at the critical points $p_{+}$or $p_{-}$. Due to the symmetry of $p_{+}$and $p_{-}$.

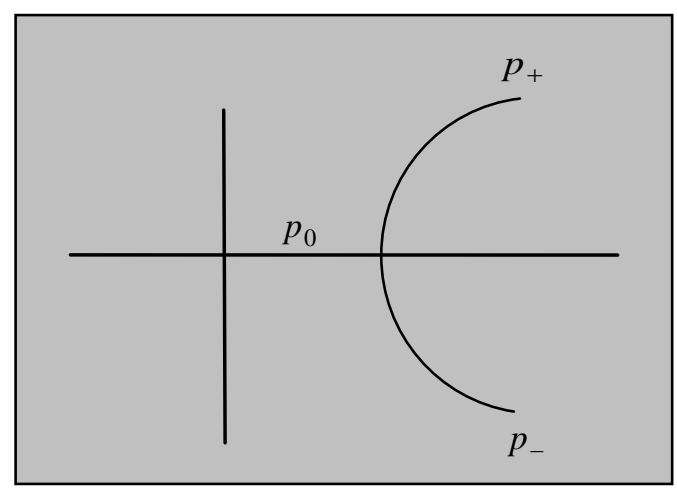

Figure 2: Sketch of the Pitchfork Bifurcation Diagram

Let us denote

$$
\begin{aligned}
q & =c-\frac{1}{3} a b+\frac{2}{27} a^{3}, \\
\Delta & =c^{2}+\frac{4}{27} b^{3}-\frac{2}{3} a b c-\frac{1}{27} a^{2} b^{2}+\frac{4}{27} a^{3} c
\end{aligned}
$$

We will use the following theorem, which enables us to find the exact roots for cubic equation (three degree).

\section{Theorem 3: (The $\Delta$-discriminate) [9]}

1- If $\Delta=0$, then has three real roots, but one is multiple:

$$
\lambda_{1}=-2 \sqrt[3]{\frac{q}{2}}-\frac{a}{3}, \quad \lambda_{2}=\lambda_{3}=\sqrt[3]{\frac{q}{2}}-\frac{a}{3}
$$

2- If $\Delta<0$, then has three different real roots: 


$$
\lambda_{k+1}=\sqrt[6]{16\left(q^{2}-\Delta\right)} \quad \cos \frac{\cos ^{-1} \frac{-q}{\sqrt{q^{2}-\Delta}}+2 \pi k}{3}-\frac{a}{3} \quad, k=0,1,2 .
$$

3- If $\Delta>0$, then has one real root $\lambda_{1}$ and two complexes conjugate roots $\lambda_{2}, \lambda_{3}$ with non-vanishing imaginary parts:

$$
\left.\begin{array}{l}
\lambda_{1}=\sqrt[3]{\frac{-q-\sqrt{\Delta}}{2}+\sqrt[3]{\frac{-q+\sqrt{\Delta}}{2}}-\frac{a}{3},} \\
\left.\lambda_{2}=-\frac{1}{2}\left(\sqrt[3]{\frac{-q-\sqrt{\Delta}}{2}}+\sqrt[3]{\frac{-q+\sqrt{\Delta}}{2}}\right)-\frac{a}{3}+i \frac{\sqrt{3}}{2}\left(\sqrt[3]{\frac{-q-\sqrt{\Delta}}{2}}-\sqrt[3]{\frac{-q+\sqrt{\Delta}}{2}}\right) \cdot\right\} \\
\lambda_{3}=-\frac{1}{2}\left(\sqrt[3]{\frac{-q-\sqrt{\Delta}}{2}}+\sqrt[3]{\frac{-q+\sqrt{\Delta}}{2}}\right)-\frac{a}{3}-i \frac{\sqrt{3}}{2}\left(\sqrt[3]{\frac{-q-\sqrt{\Delta}}{2}}-\sqrt[3]{\frac{-q+\sqrt{\Delta}}{2}}\right)
\end{array}\right\}
$$

\section{3- Main Results:}

\section{Corollary 1:}

System (2) has one negative real root $\lambda_{1}=-\beta$ at the point $p_{o}$ and also one negative real root at the point $p_{+}$.

\section{Theorem 4:}

(i ) The solution of system (2) at critical point $p_{0}$ has the following cases:

1) Asymptotically stable if $\Delta>0$ and $\operatorname{Re} \lambda_{2}<0$,

2) Unstable if $\Delta<0$ and $\lambda_{2}>0$ or $\lambda_{3}>0$,

3) Critical case if $\Delta<0$ and $\lambda_{2}=0$ or $\lambda_{3}=0$.

(ii) The solution of system (2) at critical point $p_{+}$when $\Delta>0$ has the following case:

1) Asymptotically stable if $\operatorname{Re} \lambda_{2}<0$,

2) Unstable if $\operatorname{Re} \lambda_{2}>0$,

3) Critical case if $\operatorname{Re} \lambda_{2}=0$.

\section{Proof:}

(i) When $\Delta>0$ we obtain: one real root and two complex conjugate roots (Theorem 3), and $\lambda_{1}=-\beta, \beta>0$ by corollary 1 and $\operatorname{Re} \lambda_{2}<0$ (given) then $\operatorname{Re} \lambda_{3}<0$ Since $\operatorname{Re} \lambda_{2}=\operatorname{Re} \lambda_{3}$, consequently, all roots are negative real part, hence the system (2) is asymptotically stable, while when $\Delta<0$, we get three different real roots $\lambda_{1}=-\beta$ and $\lambda_{2}>0$ or $\lambda_{3}>0$ (given) then at least one of roots is positive therefore system (2) is unstable, finally when $\lambda_{2}=0$ or $\lambda_{3}=0$ then satisfied Remark 3 therefore the system (2) is critical case the proof is completed.

(ii) since $\Delta>0$ we have one real root and two complex conjugate roots (Theorem 3), and the real root is negative at the point $p_{+}$by corollary 1 and 
1 - if $\operatorname{Re} \lambda_{2}<0$ then $\operatorname{Re} \lambda_{3}<0$ also, therefore all roots are negative real part, hence the system (2) is asymptotically stable,

2- if $\operatorname{Re} \lambda_{2}>0$ then $\operatorname{Re} \lambda_{3}>0$, consequently, real parts of two roots are positive therefore system (2) is unstable,

3- if $\lambda_{2}=0$ then $\lambda_{3}=0$ satisfied Remark 3 therefore the system (2) is critical case the proof is completed.

\section{Corollary 2:}

All three critical point $p_{0}, p_{+}$and $p_{-}$are coincide when $\sigma=2 r$.

\section{Corollary 3:}

In the critical point $p_{0}$ if:

(1) $\sigma=2 r=\beta$ then $\lambda_{1}=0, \lambda_{2}=-\beta, \lambda_{3}=-r$

(2) $\sigma=r=\beta \quad$ then $\lambda_{1}=-\beta, \lambda_{2},_{3}= \pm r= \pm \sigma=\mp \beta$

(3) $\sigma=r \quad$ then $\lambda_{1}=-\beta, \lambda_{2},_{3}= \pm r= \pm \sigma$.

\section{Theorem 5:}

The solution of system (2) at critical point $p_{+}$has the following cases

1) Asymptotically stable if $\beta>\beta_{c}$,

2) Unstable if $\beta<\beta_{c}$,

3) Critical case if $\beta=\beta_{c}$.

\section{Proof:}

Linearizing system (2) about the critical points $p_{+}$or $p_{-}$yields the following characteristic equation :

$$
f(\lambda)=\lambda^{3}+(\beta+\sigma-r) \lambda^{2}+\beta r \lambda+2 \sigma \beta(2 r-\sigma)=0
$$

Obviously, the two points $p_{ \pm}$have the same stability. Let

$$
\begin{aligned}
& a=\beta+\sigma-r \\
& b=\beta r \\
& c=2 \sigma \beta(2 r-\sigma)
\end{aligned}
$$

Then the Routh-Hurwitz criterion, (Remark 2) lead to the conclusion that the real part of the roots $\lambda$ are negative if and only if

$\beta+\sigma-r>0,2 \sigma \beta(2 r-\sigma)>0$ and $(\beta+\sigma-r) \beta r-2 \sigma \beta(2 r-\sigma)>0$

Since $a=\beta+\sigma-r$ and $\beta, \sigma$ and $r$ are $a$ positive parameters and $r<\sigma$, consequently, $a>0$ always and $c>0$ also since $\sigma \in(r, 2 r)$, when $a b>c$ we get:

$$
(\beta+\sigma-r) \beta r>2 \sigma \beta(2 r-\sigma) \Rightarrow \beta>\beta_{c}
$$

Consequently, when $\beta>\beta_{c}$ the system (2) is asymptotically stable, the proof of first condition is completed, while when $\beta<\beta_{c}$ we get $a>0$ and $c>0$ always, analogously as in proof of first case, and $a b<c$ hence one of Routh-Hurwitz conditions not satisfied, consequently the system (2) is unstable, finally when $\beta=\beta_{c}$ $a, c>0$ and $a b=c$ critical cases, the proof is completed. 
We explain our results by the following table

\begin{tabular}{|c|c|c|c|}
\hline $\begin{array}{c}\text { Relation between } \\
\sigma \sigma \text { and } 2 r\end{array}$ & $p_{o}$ & & $p_{ \pm}$ \\
\hline$\sigma>2 r$ & asymptotically stable & & not exist \\
\hline \multirow{3}{*}{$\sigma<2 r$} & \multirow{3}{*}{ unstable } & \multirow{3}{*}{ exist } & asymptotically stable if $\beta>\beta_{c}$ \\
\hline & & & unstable if $\beta<\beta_{c}$ \\
\hline & & & critical case if $\beta=\beta_{c}$ \\
\hline$\sigma=2 r$ & critical case & & not exist \\
\hline
\end{tabular}

\section{Corollary 4:}

Every hopf bifurcation and pitchfork bifurcation is critical cases but the conversely is not true.

\section{Theorem 6:}

When system (2) has critical case then this system is:

1 - Hopf Bifurcation if $\Delta>0$,

2 - Pitchfork Bifurcation if $\Delta<0$ or $\Delta=0$.

\section{Proof:}

When $\Delta>0$ we obtain: one real root and two complex conjugate roots (Theorem 3), since system (2) has critical case then

$$
\begin{aligned}
& \text { either } \lambda_{1}<0 \text { and } \operatorname{Re} \lambda_{2}=0 \quad \ldots \ldots \ldots 1 \\
& \text { or } \quad \lambda_{1}=0 \text { and } \operatorname{Re} \lambda_{2}<0 \quad \ldots \ldots \ldots . .2
\end{aligned}
$$

but equation 1 satisfied theorem 1 and remark 6, hence the system (2) has hopf Bifurcation when $\Delta>0$, the proof is complete .

When $\Delta=0$ or $\Delta<0$ we get either three real roots, but one is multiple or three different real roots (without complexes conjugate roots) and at last one of this root begin zero, therefore satisfied remark 6, hence the system (2) has Pitchfork Bifurcation when $\Delta<0$ or $\Delta=0$, the proof is complete.

\section{4- Illustrative examples:}

\section{Example 1:}

Investigates the stability and bifurcation of the following Chen System

$$
\begin{aligned}
& \dot{x}=-4 x+4 y \\
& \dot{y}=-3 x-x z+y \\
& \dot{z}=x y-5 z
\end{aligned}
$$

\section{Solution:}

\section{At critical point $p_{o}$ :}


Equation parameters this system with system (2) resulting: $\sigma=4, r=1$, By theorem (2) the Chen System is asymptotically stable since $(\sigma>2 r)$,

To compare this result with $\Delta$-discriminate theorem the characteristic equation of Chen system of the form: $\lambda^{3}+8 \lambda^{2}+23 \lambda+40=0$

and $q=\frac{448}{27}, \Delta=276, \lambda_{1}=-5, \lambda_{2},{ }_{3}=-\frac{3}{2} \pm \frac{1151}{480} i$

The Chen system is asymptotically stable at the point $p_{0}$ and also not has pitchfork Bifurcation.

\section{At critical point $p_{+}$:}

When $\sigma>2 r$, the critical point $P_{+}(\sqrt{\beta(2 r-\sigma)}, \sqrt{\beta(2 r-\sigma)}, 2 r-\sigma)$ does not exist, consequently, the Chen not has hopf bifurcation.

\section{Example 2:}

Investigates the stability and bifurcation of the following Chen System

$$
\begin{aligned}
& \dot{x}=-6 x+6 y \\
& \dot{y}=-2 x-x z+4 y \\
& \dot{z}=x y-4 z
\end{aligned}
$$

\section{Solution:}

\section{At critical point $p_{o}$ :}

Equation parameters this system with system (2) resulting: $\sigma=6, r=4$, By theorem (2) the Chen System is unstable since $(\sigma<2 r)$.

To compare this result with $\Delta$-discriminate theorem the characteristic equation of Chen system of the form: $\lambda^{3}+6 \lambda^{2}-4 \lambda-48=0$

and $q=-24, \Delta=-\frac{832}{27}, \lambda_{1}=-4, \lambda_{2}=-\frac{829}{180}, \lambda_{3}=\frac{3567}{1369}$

The Chen system unstable is at the point $p_{0}$ and also not has pitchfork Bifurcation.

\section{At critical point $p_{+}$:}

By theorem (5) the Chen System is critical case since $\beta_{c}=4, \beta=4$ and $\beta=\beta_{c}$, to compare this result with $\Delta$-discriminate theorem the characteristic equation of Chen system of the form: $\lambda^{3}+6 \lambda^{2}+16 \lambda+96=0$

and $q=80, \Delta=\frac{173056}{27}, \lambda_{1}=-6, \lambda_{2}, 3= \pm 4 i$

The Chen system is critical case at the point $p_{+}$, consequently, the Chen has hopf bifurcation.

If $\beta=5$ and $\beta_{c}=4$ then by theorem (5) we get that the system (2) asymptotically stable, to compare this result with $\Delta$-discriminate theorem the characteristic equation of Chen system of the form: $\lambda^{3}+7 \lambda^{2}+20 \lambda+120=0$ 
and $q=\frac{2666}{27}, \Delta=\frac{263440}{27}, \lambda_{1}=-\frac{1907}{285}, \lambda_{2},_{3}=-\frac{1077}{6976} \pm \frac{2061}{487} i$

The Chen system is asymptotically stable at the point $p_{+}$, consequently, the Chen has not hopf bifurcation.

We are used MATLAB to simplify computation our results at critical point $p_{+}$, and we will denote the symbols $\sigma, r, \beta, \lambda, \beta_{c}, \Delta, \lambda_{1}, \lambda_{2}, \lambda_{3}$ in the MATLAB program by $r 1, r 2, r 3, P, d$, dalta, root 1 , root 2 , root 3 , respectively.

\section{5- Program:}

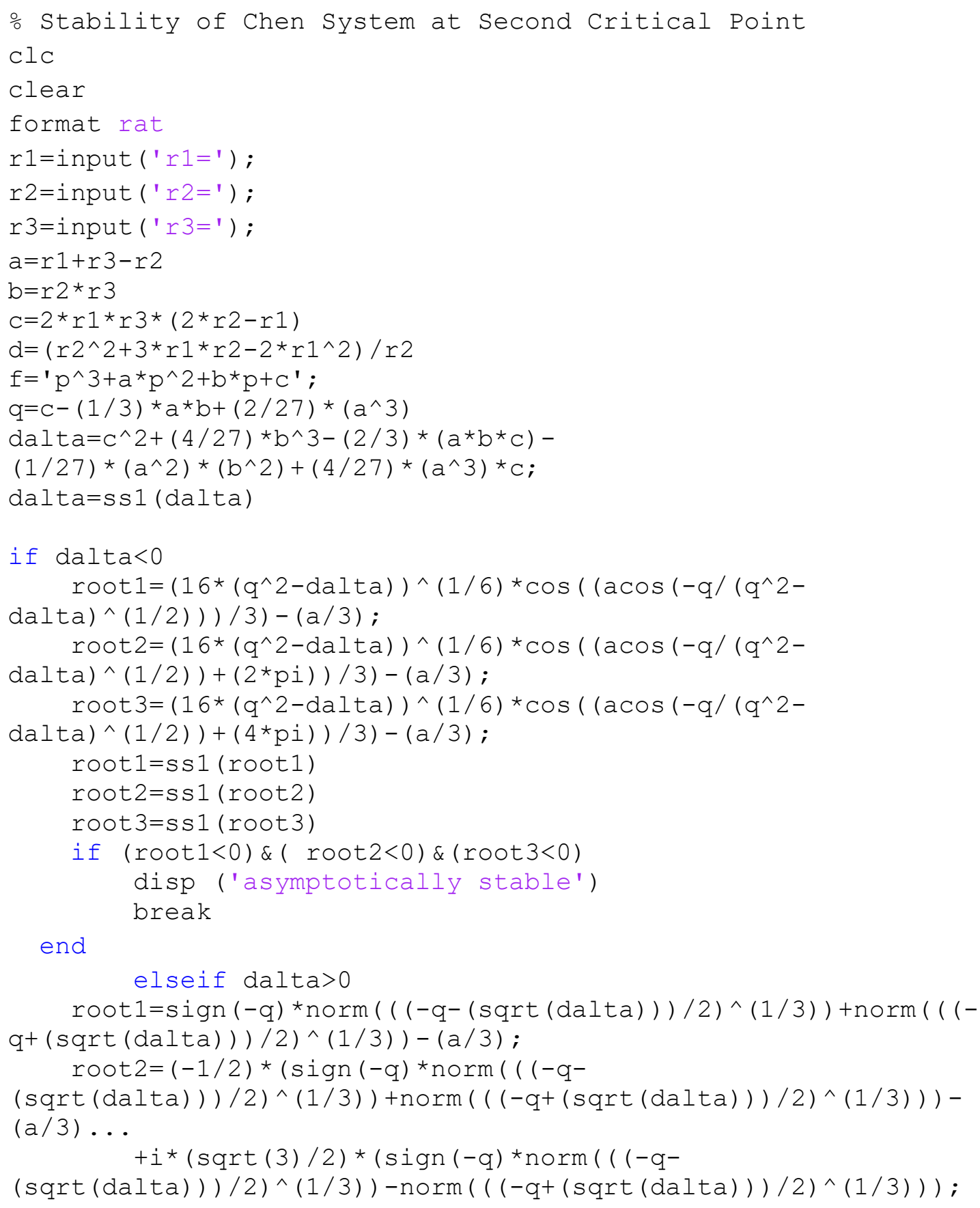




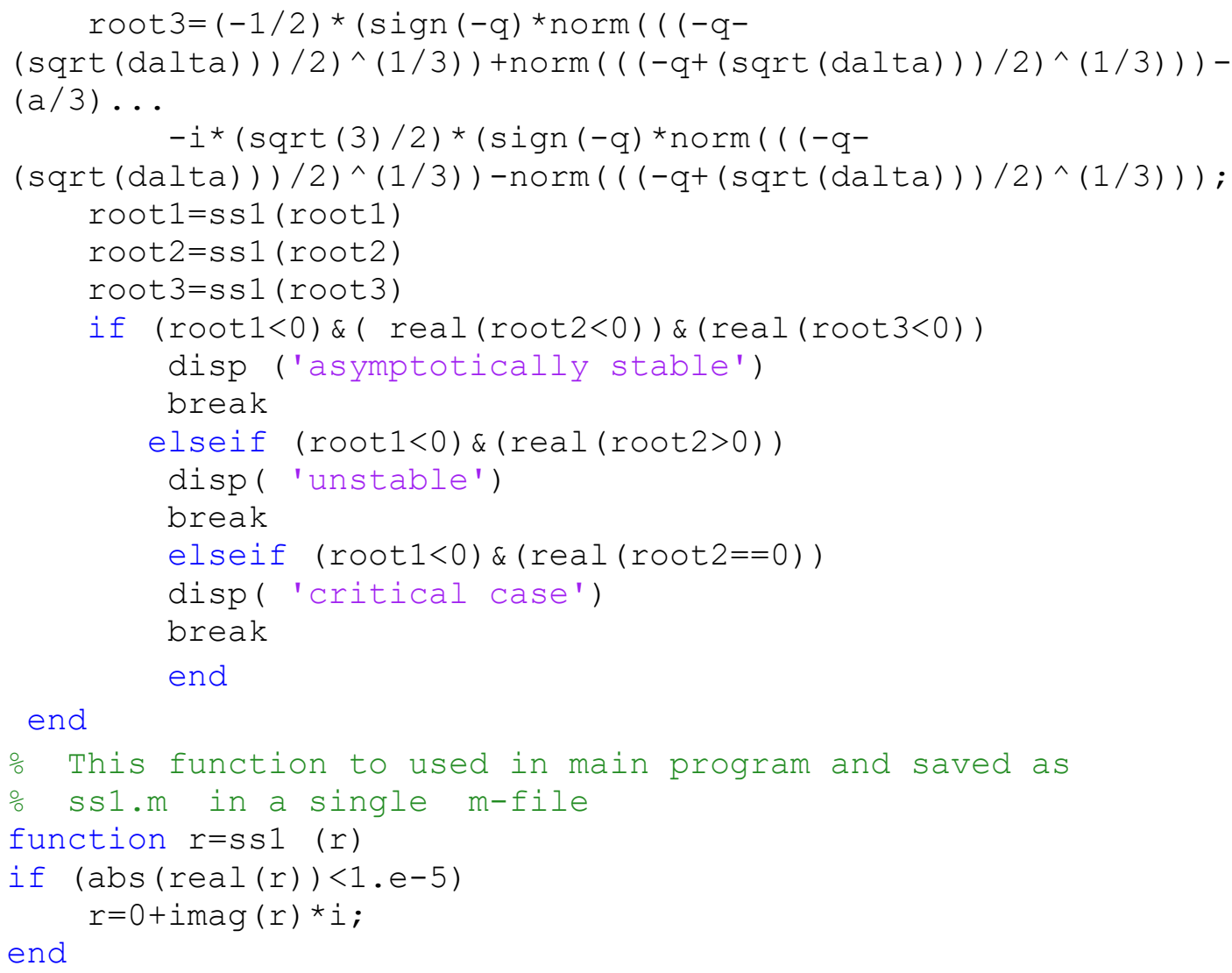

In this paper, we study the stability and bifurcation for the Chen system by using a new method which is called $\Delta$-discriminate method. and we concluded that the difference between the previous methods (Routh-Hurwitz method )and this method is depended as the previous methods on the estimate the singe of roots without found the value of this roots while in the a new method we found the exact value of this roots. 


\section{REFERENCES}

[1] Amen A.I. , Salih R.H., (2008), Limit Cycles of Lorenz System With Hopf Bifurcation, Al-Rafiden Journal of Computer Sciences and Mathematics VoI.5, No.1 , pp. 81-99.

[2] Borrelli, R.L., Coleman C.S. (1998), Differential Equations, New York., John Wiley and Sons, Inc.

[3] Cai G. , Huang J. , Tian L. , Wang Q. , (2006), A Daptive Control and Slow Manifold Analysis of a New Chaotic System , International Journal of Nonlinear Science, Vol. 2, No.1, pp. 50-60.

[4] Feng L. X., Dong C.,Y., Liang X. D., Gang Z.J., (2008), Nonlinear Dynamics and Circuit Implementation of a New Lorenz -Like Attrator, Journal of Sichuan University (Natural Science Edition) Vol. 45, No.5, pp. 1167-1173.

[5] L $\ddot{~}$ J J., Chen G., Cheng D., (2004), A New Chaotic System and Beyond: The Generalized Lorenz-Like System, International Journal of Bifurcation and Chaos, Vol.14, No.5, pp. 1507-1537.

[6] L $\ddot{~}$ J. , Chen G. ,Cheng D. ,Celikovsky S. , (2002), Bridge the Gap Between the Lorenz System and the Chen System, International Journal of Bifurcation and Chaos, Vol.12, No.12, pp. 2917-2926.

[7] L $\quad \ddot{u}$ J., Chen G., Yu X., Zhany S., (2003), Dynamical Behaviors of a Unified Chaotic Systems, Intelligent and Complex Systems, DCDIS Proceedings1, pp. 115-124.

[8] L $\ddot{~}$ J., Zhou T., Chen G., Zhang S., (2002), Local Bifurcations of the Chen System, International Journal of Bifurcation and Chaos, Vol. 12, No. 10, pp. 2257-2270.

[9] Mikołajski, J., Schmeidel, E., (2006), Comparison of Properties of Solutions of Differential Equations and Recurrence Equations with the Same characteristic Equation (On Example of Third Order Linear Equations with Constant Coefficients), Opusula Mathematics, Vol. 26, No 2, pp. 343-349.

[10] Tigan G., ( 2005), Bifurcation and Stability in a System Derived From the Lorenz System, Proceedings of the 3-rd International Colloquium "Mathematics in Engineering and Numerical Physics" Bucharest, Romania, pp. 265-272,

[11] Zhuang K., (2010), Hopf Bifurcation for a New Chaotic System, International Journal of Computational and Mathematical Sciences, Vol.4, No.8, pp.358-361. 